\title{
Neuroscience-based Nomenclature: improving clinical and scientific terminology in research and clinical psychopharmacology
}

\author{
A. B. Brühl ${ }^{1,2}$ and B. J. Sahakian ${ }^{1 *}$ \\ ${ }^{1}$ Department of Psychiatry and Behavioural and Clinical Neuroscience Institute, University of Cambridge, Cambridge, UK \\ ${ }^{2}$ Department for Psychiatry, Psychotherapy and Psychosomatics, Psychiatric Hospital, University of Zurich, Zurich, Switzerland
}

Received 4 July 2016; Revised 13 December 2016; Accepted 15 December 2016; First published online 18 January 2017

Key words: Adherence to treatment, dimensions, neuropsychopharmacology, pharmacological treatment, stigma.

In 2008, the Neuroscience-based Nomenclature $(\mathrm{NbN})$ was initiated under the leadership of the European College of Neuropsychopharmacology (ECNP), involving representatives from major bodies in the field of psychopharmacology and pharmacology: the American College of Neuropsychopharmacology (ACNP), the Asian College of Neuropsychopharmacology (AsCNP), the International College of Neuropsychopharmacology (CINP) and the International Union of Basic and Clinical Pharmacology (IUPHARM).

Psychiatrists typically prescribe 'antidepressants' and 'neuroleptic' or 'antipsychotic' drugs. The naming of the drugs is usually defined by the first intended use, and a substance usually keeps this name independently of any changing indications for use (or regulatory decisions). However, for over a decade now, things have changed: selective serotonin reuptake inhibitors (SSRIs) and other drugs initially developed for the treatment of depression are now generally also used as the main pharmacological treatments for anxiety disorders. Drugs such as quetiapine, initially developed for the treatment of psychotic symptoms, are now used in the treatment of depression, bipolar disorder, and, though off-label, for anxiety and other syndromes characterized by restlessness, anxiety, sleeping problems and agitation. Other terms such as typical $v$. atypical, first-, second- or third-generation antipsychotics or similar terms for certain groups of antidepressants were initially used to describe clinical or pharmacological features of the substances, but have since lost their precision due to the development of similar, but somewhat different drugs. For instance, atypicality which was initially seen as a categorical

* Address for correspondence: Professor B. J. Sahakian, Department of Psychiatry, University of Cambridge, Herchel Smith Building for Brain and Mind Sciences, Robinson Way, Cambridge CB2 OSZ, UK.

(Email: bjs1001@medschl.cam.ac.uk) aspect of so-called antipsychotics [a drug is either typical which means that it acts via $\mathrm{D}_{2}$ dopaminergic receptors and produces extrapyramidal side-effects, or it is atypical, i.e. acts on serotonergic $\left(5-\mathrm{HT}_{2 \mathrm{~A}}\right)$ receptors and other neurotransmitter systems and has no extrapyramidal effects] is now seen more as a dimension, which is indeed influenced by pharmacological properties such as receptor binding profiles and dissociation rate, but also by dosage and other specific properties, e.g. in the case of partial agonists (Grunder et al. 2009). In addition, psychiatric diagnosis is moving from strict categories such as affective $v$. psychotic disorders towards a more dimensional characterization of symptoms (e.g. Sahakian et al. 2010; Robbins et al. 2012) and, also supported by genetic overlaps between, for instance, schizophrenia and bipolar disorder (e.g. Moskvina et al. 2009; Purcell et al. 2009). The research domain criteria (RDoC) approach promoted by the National Institute for Mental Health (NIMH) goes even further and aims at characterizing mental disorders purely based on a descriptive matrix of neurosciencebased symptoms (Insel et al. 2010). Thus, a terminology based on an initially intended use for one circumscribed disorder or application is not in line with recent developments in pharmacology and the neuroscience of mental illness and therefore is not timely any more. Furthermore, the old terminology can make it difficult for clinicians to explain to a patient suffering from anxiety why he or she should take an antidepressant drug ('but I am not depressed, I have anxiety'), or why the depressed patient should take an antipsychotic ('I am not schizophrenic'). Therefore, patients may be confused and also suffer additional stigma from the use of the old terminology.

To overcome these problems of the current terminology, the $\mathrm{NbN}$ aims to bring a neuroscience-based and pharmacologically driven approach: drugs are described by their pharmacological mechanism of action, the neurotransmitter modulated by the drug, 
the mode of action (e.g. reuptake inhibition, enzyme inhibition, receptor action, or other modulatory effects).

There are multiple advantages of the $\mathrm{NbN}$ for many potential users: The communication between clinician and patient can be improved by not having to explain (and possibly excuse) the weaknesses of the current system and the changed use/application of medications. Research on effects of communication styles on medication adherence in mental disorders has shown that adherence can be enhanced when patients have a good understanding of their condition and the treatment options (e.g. Atreja et al. 2005). Also the communication between basic science and pre-clinical and clinical science will be improved. More and more journals such as the Journal of Psychopharmacology and the Journal Clinical Psychopharmacology and Neuroscience as well as Biological Psychiatry and its daughter journal Biological Psychiatry: Cognitive Neuroscience and Neuroimaging are all now requiring authors to use the $\mathrm{NbN}$, although in a staged fashion to enable a transition and translation between the two systems (Krystal et al. 2016a, b; Nutt \& Blier, 2016, Uchida et al. 2016), and other journals will certainly follow.

One main weakness of the $\mathrm{NbN}$ could be that it is still in development such that, for instance, drugs primarily used in child and adolescent psychiatry or in neurology are just being included. One example is the description of the mode of action of modafinil, which in the most recent version of the $\mathrm{NbN}$ is described as a dopamine (DA) reuptake inhibitor and wakefulness-promoting agent. However, the pharmacological action of modafinil is more complex, involving also a reuptake inhibiting effect for noradrenaline (Minzenberg \& Carter, 2008) as well as modulating GABA, serotonin, histamine and orexin (Scoriels et al. 2013), and besides promoting wakefulness, it also has various effects on memory, emotional and motivational processes. However, the $\mathrm{NbN}$ is explicitly designed to be extended, updated and adapted; currently, it is automatically updated twice a year.

Due to its neuroscience base the $\mathrm{NbN}$ is not only flexible and adaptable to include novel pharmacological developments, but with increasing knowledge about the neurobiology of mental disorders the factor of matching a drug to a pathophysiological mechanism will become increasingly valuable for both clinicians and scientists. Progressing this vision will require the training of a new cadre of clinician scientists (Bullmore et al. 2009; Lehner \& Insel, 2010; Insel et al. 2013), with novel programmes that focus on integrating basic and clinical neuroscience, translational medicine and novel methodologies. To achieve this, the $\mathrm{NIH}$ Neuroscience Blueprint has announced grants that target neuroscience education prior to entering university and emphasize neuroscience training at an early stage.
In conclusion, the $\mathrm{NbN}$ is a science-based approach to describing neuropsychopharmacological agents. It represents a major step that will improve scientific communication as well as the communication between clinicians and patients. We recommend that scientific publications should use the $\mathrm{NbN}$ where practical (in an intermediary period parallel to the 'old' terminology). In clinical situations, psychiatrists could begin using the mechanisms of action of drugs in addition to the 'usual' terms. This can also assist when explaining neurobiological models of disorders to patients, thereby enhancing understanding and adherence.

The $\mathrm{NbN}$ can be found here (http://nbnomenclature. org/), as well as in the Google Playstore (https://play. google.com/store/apps/details?id=il.co.inmanage.nbno menclature\&hl=en) and on Apple iTunes (https:// itunes.apple.com/us/app/nbn-neuroscience-based-nom enclature/id927272449? $\mathrm{mt}=8$ ). To help authors to 'translate' the usual nomenclature for publications, the $\mathrm{NbN}$ also offers a glossary for download (http:// nbnomenclature.org/_inc/layout/save_pdf.inc.php?file= NbN_Glossary.pdf\&action=download_pdf).

\section{Declaration of Interest}

B.J.S. is a member of the ACNP Nomenclature Workgroup.

\section{References}

Atreja A, Bellam N, Levy SR (2005). Strategies to enhance patient adherence: making it simple. Medscape General Medicine 7, 4-4.

Bullmore E, Fletcher P, Jones PB (2009). Why psychiatry can't afford to be neurophobic. British Journal of Psychiatry 194, 293-295.

Grunder G, Hippius H, Carlsson A (2009). The 'atypicality' of antipsychotics: a concept re-examined and re-defined. Nature Reviews Drug Discovery 8, 197-202.

Insel TR, Cuthbert BN, Garvey MJ, Heinssen R, Pine DS, Quinn K, Sanislow CA, Wang P (2010). Research Domain Criteria (RDoC): toward a new classification framework for research on mental disorders. American Journal of Psychiatry 167, 748-751.

Insel TR, Voon V, Nye JS, Brown VJ, Altevogt BM, Bullmore ET, Goodwin GM, Howard RJ, Kupfer DJ, Malloch G, Marston HM, Nutt DJ, Robbins TW, Stahl SM, Tricklebank MD, Williams JH, Sahakian BJ (2013). Innovative solutions to novel drug development in mental health. Neuroscience \& Biobehavioral Reviews 37, 2438-2444.

Krystal JH, Abi-Dargham A, Barch DM, Bullmore ET, Carter CS, Geschwind DH, Harrison PJ, Nestler EJ, Stein MB (2016a). Biological psychiatry and biological psychiatry: cognitive neuroscience and neuroimaging adopt neuroscience-based nomenclature. Biological Psychiatry 80, 2-3.

Krystal JH, Abi-Dargham A, Barch DM, Bullmore ET, Carter CS, Geschwind DH, Harrison PJ, Nestler EJ, Stein MB 
(2016b). Biological psychiatry and biological psychiatry: cognitive neuroscience and neuroimaging adopt neuroscience-based nomenclature. Biological Psychiatry: Cognitive Neuroscience and Neuroimaging 1, 300-301.

Lehner T, Insel T (2010). Psychiatric education in the genomic era. Academic Psychiatry 34, 87-89.

Minzenberg MJ, Carter CS (2008). Modafinil: a review of neurochemical actions and effects on cognition. Neuropsychopharmacology 33, 1477-1502.

Moskvina V, Craddock N, Holmans P, Nikolov I, Pahwa JS, Green E, Owen MJ, O'Donovan MC (2009). Gene-wide analyses of genome-wide association data sets: evidence for multiple common risk alleles for schizophrenia and bipolar disorder and for overlap in genetic risk. Molecular Psychiatry 14, 252-260.

Nutt DJ, Blier P (2016). Neuroscience-based Nomenclature $(\mathrm{NbN})$ for Journal of Psychopharmacology. Journal of Psychopharmacology 30, 413-415.
Purcell SM, Wray NR, Stone JL, Visscher PM, O'Donovan MC, Sullivan PF, Sklar P, Ruderfer DM, McQuillin A,

Morris DW (2009). Common polygenic variation contributes to risk of schizophrenia and bipolar disorder. Nature 460, 748-752.

Robbins TW, Gillan CM, Smith DG, de Wit S, Ersche KD (2012). Neurocognitive endophenotypes of impulsivity and compulsivity: towards dimensional psychiatry. Trends in Cognitive Sciences 16, 81-91.

Sahahian BJ, Malloch G, Kennard C (2010). A UK strategy for mental health and wellbeing. Lancet 375, 1854-1855.

Scoriels L, Jones PB, Sahakian BJ (2013). Modafinil effects on cognition and emotion in schizophrenia and its neurochemical modulation in the brain. Neuropharmacology 64, 168-184.

Uchida H, Yamawaki S, Bahk W-M, Jon D-I (2016).

Neuroscience-based Nomenclature $(\mathrm{NbN})$ for clinical psychopharmacology and neuroscience. Clinical Psychopharmacology and Neuroscience 14, 115-116. 